\title{
Rate-Compatible Distributed Arithmetic Coding
}

\author{
Marco Grangetto, Member, IEEE, Enrico Magli, Senior Member, IEEE, Roberto Tron, \\ and Gabriella Olmo, Senior Member, IEEE
}

\begin{abstract}
Distributed arithmetic coding has been shown to be effective for Slepian-Wolf coding with side information. In this letter, we extend it to rate-compatible coding, which is useful in presence of a feedback channel between encoder and decoder. The performance loss with respect to the original version is negligible.
\end{abstract}

Index Terms-Distributed source coding, arithmetic coding, Slepian-Wolf coding, Wyner-Ziv coding, compression.

\section{INTRODUCTION}

$\mathbf{T}$ HE theory of distributed source coding (DSC) proves that separate lossless encoding of two or more correlated data sources is optimal, provided that the sources are jointly decoded. A classical situation encompasses a first source, or side information, which is subject to standard encoding, and a second one that is conditionally encoded at a rate lower than its entropy. Practical DSC coders typically represent the source using the syndrome or parity bits of a suitable channel code of given rate, e.g. trellis codes, turbo codes and low-density parity-check codes.

In [1] a Slepian-Wolf coder is proposed based on a modification of the arithmetic coder (AC). The scheme, named distributed arithmetic coder (DAC), encodes a binary source at a rate smaller than its entropy by allowing the intervals to overlap and generating an ambiguous codeword. A soft joint decoder exploits knowledge of the side information to decode the source. DAC has been shown to work well also for short block lengths, representing a good solution for image or video coding.

To take advantage of a feedback channel between the encoder and the decoder, the DAC principle has to be revisited to provide a rate-compatible system, similarly to what can be done with turbo [2] and low-density parity-check (LDPC) codes [3]. The algorithm proposed in this letter builds upon the DAC, and proposes a rate-compatible DAC-based algorithm. In particular, the DAC decoding procedure is modified to allow the transmission on the feedback channel of some extra bits upon decoder request, in case this latter encounters a decoding ambiguity. This allows to reduce the residual bit-error rate (BER), up to error-free decoding.

Manuscript received April 24, 2008. The associate editor coordinating the review of this letter and approving it for publication was V. Stankovic.

M. Grangetto is with the Department of Informatics, Università degli Studi di Torino, Torino, Italy (e-mail: marco.grangetto@unito.it).

E. Magli and G. Olmo are with the Department of Electronics, Politecnico di Torino, Torino, Italy (e-mail: \{enrico.magli, gabriella.olmo\}@polito.it).

$\mathrm{R}$. Tron is with the Vision Lab Center for Imaging Science, Johns Hopkins University, Baltimore, MD USA (e-mail: tron@cis.jhu.edu).

Digital Object Identifier 10.1109/LCOMM.2008.080645.

\section{DistRIBUTED ARITHMETIC CODING}

Let $\mathbf{X}=\left[X_{0}, X_{1}, \cdots X_{N-1}\right]$ be a length- $N$ binary sequence to be encoded, with probability distribution $p_{0}=$ $P\left(X_{i}=0\right)$ and $p_{1}=P\left(X_{i}=1\right), i=0, \cdots, N-1$, and let $\mathbf{Y}=\left[Y_{0}, Y_{1}, \cdots Y_{N-1}\right]$ be a correlated side information binary symbol sequence. In classical $\mathrm{AC}$, the source symbols $X_{i}$ are iteratively mapped onto sub-intervals of $[0,1)$, whose lengths are proportional to $p_{0}$ and $p_{1}$. The codeword $\mathbf{C}_{X}$ representing $\mathrm{X}$ consists in a binary number lying in the final interval $I$, whose average length is $L=-\log _{2}|I|$ bits, where $|I|$ is the length of $I$.

In DAC [1], the interval lengths are taken proportionally to the modified probabilities $\widetilde{p}_{0}=\alpha_{0} p_{0}$ and $\widetilde{p}_{1}=\alpha_{1} p_{1}$, with $\alpha_{j} \geq 1$ for $j=0,1$. The sub-intervals are allowed to partially overlap in order to fit the $[0,1)$ interval. As a consequence, the decoder will typically be unable to decode the source unambiguously without knowledge of the side information. The overlapping leads to a larger final interval, and hence a shorter codeword; $\alpha_{j}$ is chosen such that the codeword length matches the desired bit-rate. We describe the ambiguity defining a ternary symbol $\tilde{X}_{i} \in(0,1, a), i=0, \cdots, N-1$ that represents the decoding of the $i$-th input symbol; $a$ represents a decoding ambiguity corresponding to $\mathbf{C}_{X}$ lying in an overlapped region.

The DAC decoding process can be formulated as a symboldriven sequential search along a proper decoding tree, where each node represents a state of the sequential arithmetic decoder. When the $i$-th input symbol is decoded, if $\tilde{X}_{i}=a$ the decoder performs a branching. Since $a$ could be equal to either 0 or 1 , two alternative paths are stored in the decoding memory, corresponding to the two alternative symbols $X_{i}=0$ and $X_{i}=1$ that could be decoded at this step. For each new state, the associated branch metric is updated, and the corresponding interval is selected for next iteration; in particular, the Maximum A Posteriori (MAP) metric $P\left(\mathbf{X} \mid \mathbf{C}_{X}, Y\right)$ is employed. In order to reduce complexity, after decoding a new input symbol, the decoder keeps only the $M$ paths with the best partial metric, and prunes the others; this is done using the $M$-algorithm. More details on the DAC encoding and decoding procedures can be found in [1], [4].

\section{RATE-COMPATIBLE DAC}

Due to the limited available memory, at each step the DAC decoder is able to store only a limited number $M$ of candidate sequences. As a consequence, it may happen that the correct path is discarded, as it exhibits a partial metric that is worse than that of other paths; in this case, decoding errors may occur. In particular, errors may stem from erroneous decisions regarding decoded bits, when the received codeword lies in an 
ambiguous probability interval (i.e. $\tilde{X}_{i}=a$ ) in one of the $M$ active paths. In this situation, the decoding process may be helped if the encoder passes on to the decoder those bits that turn out to be ambiguous during the decoding process (named feedback bits - FB). Upon detection of decoding failure, e.g. by using a cyclic redundancy check code, the decoder can have one or more FBs sent over the feedback channel, and refine its first decoding attempt, possibly correcting the erroneous choices. To this end, it is necessary that the encoder and the decoder agree on a list of ambiguous symbols for which FBs may be needed. The ambiguous symbols depend on both $\mathbf{X}$ and $\mathbf{Y}$. An explicit list would require a large amount of extra rate, as well as explicit communication between $\mathbf{X}$ and $\mathbf{Y}$. On the other hand, the encoder can mimic the decoder operations and create a list of the input symbols that will be recognized as ambiguous by the decoder. In doing so, it exploits its knowledge of the original sequence, always taking correct decisions at branching nodes. This can be done in several ways; three options have been considered in this letter, namely: direct ordering, inverse ordering, hybrid ordering.

\section{A. Direct ordering}

1) Encoder: In direct ordering, the FBs are inserted in a list (called the $F B$ list) following the normal forward ordering of the encoding and decoding procedures. The generation of the FB list $f_{D}(\ell)$ at the encoder, containing $L_{F}$ bits, can be summarized as follows.

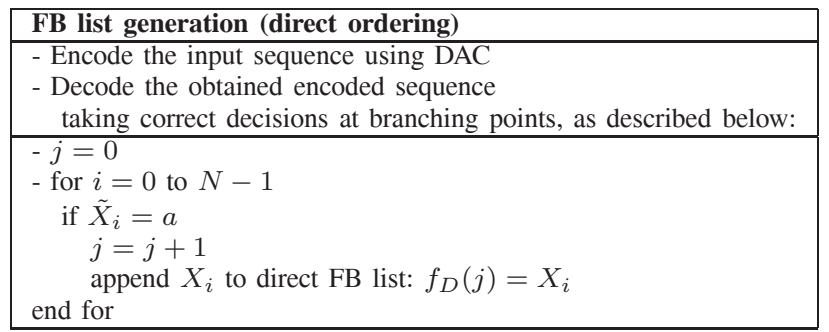

It is worth noticing that only the values of the FBs, not their positions, need to be transmitted to the decoder.

2) Decoder: When the decoder is unable to reconstruct the source using the received DAC codeword, FBs are extracted from the list and sent to the decoder upon request. With direct ordering, the decoding operations are very simple. DAC decoding is performed as usual; every time an ambiguous symbol is encountered, an FB is used to drive the correct decoding branch, until all the available FBs have been used; then, usual DAC decoding is performed to deal with further branchings.

However, it has been found that decoding errors tend to occur mostly at the end of the sequence [4]. In fact, correct decoding happens because wrong paths that diverge from the correct one at the beginning of the sequence, are likely to be discarded, as the metric of the correct path asymptotically tends to get dominant. Therefore, the direct ordering is suboptimal in terms of additional rate, in that the first transmitted FBs are less useful, as they do not refer to the most critical branches.

\section{B. Inverse ordering}

1) Encoder: Sending the FBs in inverse order (i.e., starting from those referring to the last branching events) is more efficient if not all the FBs are transmitted, as these latter are more prone to decoding errors. The generation of the FB list in inverse order $f_{I}(\ell)$, follows a procedure identical to direct ordering, except that the bits are transmitted in inverse order, i.e. $f_{I}(\ell)=f_{D}\left(L_{F}-\ell\right)$.

2) Decoder: Inverse ordering makes the decoder design more complex. In fact, it is possible that not all the $M$ sequences that are retained by the decoder stem from decoder branching decisions on ambiguous bits that match the transmitted FBs. In case a sequence (i.e. a path) is not congruent with the FBs, it should be removed from the decoder list, and another sequence, previously disregarded due to the lack of decoding memory, should be resumed. In order to keep track of which sequences have been discarded by the $M$-algorithm and which ones do not match the FBs, it is necessary to store the whole decoding tree in the decoder memory. The modified decoding process can then be summarized as follows:

1) Decode the whole sequence and store the whole decoding tree. At the end of the decoding procedure, the $M$ outmost leaves of the decoding tree represent the set of candidate sequences, among which the one with the best metric will be selected.

2) Backward-check the decoding tree using the FBs.

3) Clear the decoding sequences that do not match the FBs.

4) Revisit the decoding tree and resume already discarded branches.

5) Select the sequence with the best decoding metric.

The backward-check verifies if the sequences selected by the decoder match the FBs at the branching points. As the FBs are transmitted in inverse order, it is necessary to scan the decoding tree from the leaves towards the root. Backwardcheck is implemented by means of a recursive procedure. Starting from a leaf of the tree, it is verified whether the branch leading to that leaf matches the FBs. In case it does not, that leaf must be pruned; otherwise, the control step moves on to the parent node, and so forth until all the FBs have been scanned. In case a sequence is not compliant with the FBs, it is removed from the decoding memory, and the related leaf is pruned from the decoding tree.

In order to make the memory management more efficient, and to speed up the algorithm execution, it is necessary to cancel those paths that have already been completely explored, and that cannot lead to decoding solutions compatible with the information contained in the FB list. This is done by means of the clearing step. It is a recursive procedure that visits each node in the tree. If both children nodes have already been checked, and do not match the FBs, that node is pruned from the tree. The branches are pruned progressively from the leaves towards the root, avoiding to prune any node that can lead to solutions not already explored.

In case one or more sequences have been cleared, sequences previously discarded due to finite $M$ can be resumed. The restarting step visits the decoding tree in depth, and restores some of those branches that had already been discarded by 
TABLE I

AVERAGE BIT-RATE (BPS) NECESSARY FOR ERROR-FREE DECODING

\begin{tabular}{|c|c|}
\hline Initial bit-rate & Average bit-rate \\
\hline$p_{0}=0.5, p=0.1102, N=100$ \\
\hline 0.53 & 0.58 \\
0.56 & 0.59 \\
0.59 & 0.61 \\
\hline \multicolumn{2}{|c|}{$p_{0}=0.8, p=0.23, N=200$} \\
\hline 0.57 & 0.64 \\
0.61 & 0.62 \\
\hline
\end{tabular}

the $M$-algorithm. The selection of the visiting order is done in a greedy manner, starting from the children nodes with better metric. When visiting a node whose children have not been explored yet, the decoding and the clearing passes are repeated starting from that node. After that, the visit of nodes for the restarting procedure continues recursively on the just created path. The procedure stops when all possible sequences have been verified, or the decoding memory is full. In order to terminate the decoding, all the solutions found before and after the restarting step are compared, and that with the best accumulated metric is selected as the final solution.

As a final comment, it should be noted that, for both direct and inverse ordering, correct decoding of the whole sequence is ensured if all the FBs are transmitted and $L_{F}$ is known at the decoder.

\section{Hybrid ordering}

In order to blend the advantages of the two discussed approaches, a hybrid ordering has also been considered. The first $K$ FBs are transmitted in inverse order, whereas the others are transmitted in direct order. In this way, a trade off between rate performance and decoding complexity can be achieved.

The decoder starts with inverse ordering as long as it has used the first $K$ FBs. This yields a set of decoder states that are compatible with those FBs. Then, it uses the direct ordering decoding procedure, which selects one out of the available candidates, identifying the decoder best path and hence allowing to output the decoded sequence.

\section{EXPERIMENTAL RESULTS}

We use an AC machinery in 32-bit fixed-point implementation with interval rescaling. $N$ samples of a binary memoryless source $\mathbf{X}$ have been generated. The side information $\mathbf{Y}$ is obtained as the output of a binary symmetric channel with input $\mathbf{X}$ and transition probability $p$. In each simulation and for each data block, the source data are compressed using DAC at a selected initial bit-rate, without using the FBs. Then, the FBs are used one at a time, until the number of decoding errors for that block is zero. In all simulations the hybrid algorithm with $K=15$ has been employed.

Simulations have been run using 1000 blocks with $N=$ $100, p_{0}=0.5, p=0.11$ (corresponding to $H(\mathbf{X} \mid \mathbf{Y})=0.50$ ). Tab. I reports the average bit-rate for error-free decoding; note that, unlike fixed-rate coding, these variable-rate simulations converge very quickly with a small number of trials. As can be seen, it is convenient to take an initial bit-rate that slightly exceeds the Slepian-Wolf bound; in this case, error-free decoding is achieved at a rate about $0.07 \mathrm{bps}$ larger than the bound. For comparison, a non rate-compatible DAC achieves error-free decoding at a rate equal to $0.58 \mathrm{bps}$. This is obtained averaging 1000 trials in which each sequence is reencoded with DAC at increasing rate up to error-free decoding; while this procedure does not represent a practical solution, it is a good benchmark for the rate-compatible algorithm. These results show that the performance loss incurred for rate compatibility is negligible. The rate used by the decoder to request more bits is equal to the difference between the average and initial bit-rates, and can be made small by sending multiple FB bits at a time.

Another simulation has been performed using 100 data blocks with $N=200, p_{0}=0.8$ and $p=0.23$ (corresponding to $H(\mathbf{X} \mid \mathbf{Y})=0.58)$. Error-free decoding is achieved at a rate about 0.06 bps larger than the bound. For comparison, the non rate-compatible DAC achieves a rate of $0.61 \mathrm{bps}$.

For the sake of comparison, we have also simulated a system based on rate-compatible LDPC codes such as in [3], and on turbo codes such as in [2]. For the LDPC code, the only block length available in the software implementing [3] is $N=396$. For the turbo code, we use block lengths of $N=100$ and $N=200$ with S-random interleaving. For $p_{0}=0.5$ and $p=0.11$, the average bit-rate for error-free decoding turns out to be 0.59 bps for irregular LDPC codes and 0.62 for regular codes. Under the same conditions, the turbo code with $N=100$ achieves 0.73 bps. For $N=200$, $p_{0}=0.8$ and $p=0.23$, the irregular and regular LDPC codes achieve 0.67 and 0.69 bps respectively, while the turbo code achieves $0.99 \mathrm{bps}$; the poor performance of turbo codes for asymmetric input distribution has also been noted in [4]. Hence, for small block lengths, the rate-compatible DAC outperforms turbo and LDPC codes. However, as noted in [4], unlike turbo and LDPC codes, the DAC performance does not improve significantly increasing the block length.

\section{CONCLUSiOnS}

In this letter we have introduced a rate-compatible version of DAC. It has been shown that the proposed algorithm outperforms LDPC and turbo codes, and that the performance loss of the rate-compatible with respect to the standard DAC is negligible. This makes DAC suitable for applications where a feedback channel is available for incremental transmission of a source with side information is available at the decoder.

\section{REFERENCES}

[1] M. Grangetto, E. Magli, and G. Olmo, "Distributed arithmetic coding," IEEE Commun. Lett., vol. 11, no. 11, pp. 883-885, Nov. 2007.

[2] B. Girod, A. Aaron, S. Rane, and D. Rebollo-Monedero, "Distributed video coding," Proc. IEEE, Special Issue on Advances in Video Coding and Delivery, pp. 71-83, Jan. 2005.

[3] D. Varodayan, A. Aaron, and B. Girod, "Rate adaptive codes for distributed source coding," Signal Processing, vol. 86, no. 11, pp. 31233130, Nov. 2006.

[4] M. Grangetto, E. Magli, and G. Olmo, "Distributed arithmetic coding for the asymmetric Slepian-Wolf problem," IEEE Trans. Signal Processing (submitted). 